Projets

de Paysage

\section{Projets de paysage}

Revue scientifique sur la conception et l'aménagement de l'espace

$8 \mid 2012$

Les concepteurs de jardins et de parcs japonais

\title{
Croisements paysage/danse/musique : écritures entre composition et improvisation
}

Landscape/Dance/Music Junctions: Writings between composition and Improvisation

\section{Mathilde Christmann}

\section{(2) OpenEdition}

\section{Journals}

Édition électronique

URL : http://journals.openedition.org/paysage/15564

DOI : 10.4000/paysage.15564

ISSN : 1969-6124

Éditeur :

École nationale supérieure du paysage de Versailles-Marseille, Institut national des sciences appliquées Centre Val de Loire - École de la nature et du paysage, École nationale supérieure d'architecture et de paysage de Bordeaux, École nationale supérieure d'architecture et de paysage de Lille, Agrocampus Angers

\section{Référence électronique}

Mathilde Christmann, « Croisements paysage/danse/musique : écritures entre composition et improvisation », Projets de paysage [En ligne], 8 | 2012, mis en ligne le 14 juillet 2012, consulté le 11 mars 2021. URL : http://journals.openedition.org/paysage/15564 ; DOI : https://doi.org/10.4000/ paysage. 15564

Ce document a été généré automatiquement le 11 mars 2021.

Projets de paysage 


\title{
Croisements paysage/danse/ musique : écritures entre composition et improvisation
}

\author{
Landscape/Dance/Music Junctions: Writings between composition and
}

Improvisation

Mathilde Christmann

1 Nous mettrons ici le paysage en dialogue avec deux autres disciplines, la danse et la musique, par le biais d'écritures développées par les paysagistes, les compositeurs et les chorégraphes pour donner naissance à leur esthétique et faire évoluer leurs processus de conception.

2 Ces trois disciplines du paysage, de la danse et de la musique trouvent un sol commun dans le rapport qu'entretiennent leurs écritures au mouvement. Le mouvement contenu dans le paysage, la danse et la musique en tant que présences vivantes, constitue leur essence : le paysage comme somme de vie et de forces en mouvement, la danse comme présence pure des corps se mouvant dans l'espace, et la musique au déroulement temporel de rythmes, de mélodies et d'harmonies dont les mouvements s'interpénètrent dans des gestuelles vocales, instrumentales et théâtrales. Les conditions d'émergence du paysage, de la danse et de la musique se situent intrinsèquement dans un espace-temps en mouvement. Mais davantage : puisqu'elles sont créatrices de formes artistiques, les créations du paysagiste, du compositeur et du chorégraphe s'élaborent dans un processus de conception qui fait intervenir l'écriture, aussi bien pour engager des idées que pour les communiquer :

... écritures sous forme de plans, coupes, schémas, grilles, diagrammes, mots, dessins, notes, portées, tracés...

... écritures qui peuvent faire signes, symboles, langages, graphies...

Dès lors que l'on croise nos trois disciplines et leur histoire scripturale se joue une pluralité de modes et de présences qui témoignent de l'acte créateur dans ses rapports à l'espace et au temps et sont susceptibles de s'éclairer l'une l'autre. Leur rapport au 
mouvement engendre la pensée d'une écriture qui doit réfléchir sur le contenu «mouvant» de ce qu'elle met en œuvre sur le papier. Comment inscrire une présence mouvante, une spatialité rythmée par le temps ? Quelle matière tracer?

Cette dernière question à laquelle tenter de répondre assemble deux concepts - matière et tracement - empruntés à deux philosophes étudiant pour l'un la danse, et pour l'autre le paysage.

5 Le premier, Frédéric Pouillaude, s'inscrit dans le contexte de la danse, où il explore la possibilité pour le chorégraphe de mettre en œuvre une " écriture par matière »; voici ce qu'y signifie la matière: "Matière est un terme qui fait l'objet en danse contemporaine d'une riche ambiguiité. Il désigne simultanément le produit brut de l'improvisation - matière attendant d'être mise en forme -, et l'objet idéal de la composition - matière ouvrant, tel un thème musical, un espace de variation et d'improvisation seconde ${ }^{1}$." La matière en danse entremêle donc les processus d'improvisation et de composition qui président à la création.

6 Le second philosophe, Jean-Marc Besse, enseigne le paysage et l'histoire de la géographie. Dans un article dans lequel il s'intéresse à la cartographie comme possibilité de construction et d'invention, il conclut par ces mots: "Mais il faut aller peut-être au plus radical encore, au tracé et au geste du tracement initial, et conclure : c'est le tracement qui rend possible, c'est le tracement qui est la condition de possibilité de tout le reste en général. Ce mouvement vers l'avant est celui de la pensée tout entière ${ }^{2}$. » L'acte de tracer est considéré par Jean-Marc Besse comme une condition à l'apparition de la pensée.

7 L'idée d'une écriture qui serait tracement d'une matière permet de féconder le dialogue des trois disciplines convoquées ici en regard. En effet, l'écriture telle qu'elle s'entend en ce sens se trouve en position de notation «élargie ", c'est-à-dire qu'elle outrepasse le caractère symbolique qui lui est acquis de manière générale en tant que langage, pour considérer plus largement les jeux de composition, d'improvisation et les présences gestuelles qui ont cours lorsqu'elle est pratiquée par la pensée en création.

8 Ainsi, lorsque nous emploierons le mot « écriture ", il sera désormais implicitement fait référence à l'ensemble de ces tensions qui n'ont finalement pour quête que la poursuite du mouvement.

9 Ce tracement d'une matière est abordé ici à travers trois dispositifs créatifs singuliers qui, chacun à leur manière, cherchent par l'écriture de la partition à rendre compte d'une tension entre processus de composition et processus d'improvisation. La composition est fortement ancrée dans l'inconscient collectif comme écriture - le terme nous fait aisément imaginer notre " compositeur » de musique assis à sa table et complétant ses portées. Cette attitude de création est proprement liée à la volonté d'organiser et de structurer sa pensée par l'écriture, celle-ci fixant des formes que l'on peut ensuite regarder (la peinture ou le jardin par exemple), exécuter (la musique), lire (le roman), etc. Il en va autrement de l'improvisation, qui qualifie inconsciemment un acte du présent, un geste né d'une spontanéité, synonyme d'invention. Dans le champ artistique, l'improvisation a des racines très anciennes, notamment dans la culture dite "populaire ", mais elle n'a été redécouverte et réexploitée qu'assez récemment, à partir des années 1950 . Son lien à l'écriture semble insaisissable - ici, c'est le jazzman qui nous apparait, occupé du seul flot changeant que déverse son instrument. 
10 Et pourtant certaines œuvres, dans leur singularité, savent nous dire beaucoup du lien essentiel qu'entretient l'écriture avec la composition et avec l'improvisation. Ces deux modalités d'écriture sont en fait assez proches et tout à fait complémentaires, ce que nous allons explorer à travers trois dispositifs artistiques relevant chacun d'un des trois champs étudiés :

- pour le paysage : la pensée des cycles RSVP du paysagiste américain Lawrence Halprin et son illustration dans quelques-uns de ses projets;

- pour la musique: la création de Zwei-Mann-Orchester, pièce orchestrale du compositeur argentin Mauricio Kagel ;

- pour la danse : l'écriture inspirée de la notation Laban développée par la chorégraphe française Myriam Gourfink, ici à travers la pièce Les Temps tiraillés.

11 Nous envisagerons ensuite les croisements opérables entre ces trois œuvres et les questions qui se posent à l'occasion d'une telle recherche interdisciplinaire s'adressant au paysagiste.

\section{Lawrence Halprin}

Lawrence Halprin est un paysagiste américain, né en 1916 et mort en 2009. Avec sa femme Anna Halprin, danseuse et chorégraphe, ils travaillent à ouvrir les possibilités créatives de chacun et mettent au point les cycles RSVP, qu'ils appliqueront chacun à leur discipline: le paysage et l'aménagement des villes pour Lawrence, et la postmoderne dance - courant avant-gardiste de danse contemporaine aux États-Unis pour Anna. Ils habitent et investissent la Côte ouest-américaine, où ont lieu la très grande majorité de leurs projets. Lawrence Halprin fonde son agence de paysage en 1949 à San Francisco, «Halprin \& Associates ». Il se déclarait lui-même "paysagiste écologiste ", ce qui à cette époque témoignait d'une grande attention portée sur les paysages « naturels » en tant que conjonction de vies et de mouvements s'entrecroisant (vie biologique, vie humaine, vie spirituelle, vie tellurique, etc.). Halprin est particulièrement fasciné par les processus et les forces de la nature qui lui semblent nécessairement liés aux activités humaines, de façon "organique ", dans les formes même de vie que les uns et les autres organisent.

13 Sa philosophie de vie, qu'il s'efforce d'appliquer à ses projets, est résolument ancrée dans son époque, les années 1960, marquée par la quête de liberté et d'une vie alternative tournée vers la communauté. Lawrence et Anna Halprin prônent une créativité individuelle vivante et la possibilité pour chacun de s'exprimer par l'art. Ils s'intéressent aux liens et aux échanges pacifiés au sein des communautés et réfléchissent sur les libertés, tant individuelles que collectives, que l'art peut engendrer.

14 Cette éthique de vie, liée à leurs activités professionnelles, va engager les Halprin à mettre en œuvre un grand nombre de workshops pluridisciplinaires mêlant étudiants et non-étudiants, danseurs, architectes, graphistes, poètes ou sculpteurs. Ces ateliers sont basés sur une invention du paysagiste - du moins dans sa formulation : les cycles RSVP, qui résument en quatre lettres le processus créatif :

- $\mathrm{R}$ pour les ressources, ressources en anglais, c'est-à-dire les matériaux, tant physiques qu'humains, qui permettent de se fixer des objectifs quant à l'activité artistique entreprise ;

- $\mathrm{S}$ pour les partitions, scores, qui décrivent les processus menant à l'exécution ; 
- V pour la valuaction, contraction anglaise des mots transparents evaluation et action, qui analyse l'action une fois qu'elle a eu lieu et permet de prendre des décisions ;

- $\mathrm{P}$ pour l'exécution, performance - ce mot anglais désignant simplement le fait de faire, de mettre en œuvre, d'accomplir quelque chose - qui, par l'action, donne son style au processus.

Un système appelé Motation par exemple caractérise le début des recherches d'Halprin. Ce mot inventé par le paysagiste, intraduisible en français, est la contraction des termes anglais motion (mouvement) et notation (notation). Il s'agit dans ces partitions de mettre en relation le mouvement des gens et celui de leur environnement pour montrer comment ils s'affectent l'un l'autre et forment chorégraphie. Halprin s'est essayé à l'un de ce système pour le réaménagement de la Nicollet Avenue en 1968 à Minneapolis.

Il a ensuite mis en place d'autres formes partitionnelles comme les City Map dans le contexte d'ateliers de sensibilisation à la ville avec des élus, des habitants, des designers d'espace, etc. En 1973 par exemple, à Cleveland, dans le cadre du renouveau urbanistique de la ville, 30 participants se sont vu distribuer une partition pour arpenter la ville et influer sur ses projets urbains à venir. Chacun passait dans les mêmes lieux avec des consignes à respecter, mais à différents moments de la journée, de façon à éprouver seul l'espace le temps d'une journée puis de mettre ces expériences en commun. Les participants étaient ensuite invités à travailler en groupe pour transcrire graphiquement leurs ressentis sous forme de dessins, de croquis, de plans et de textes.

19 Habitant de la Côte ouest-américaine, Halprin avait pour habitude d'organiser avec sa femme des workshops d'été dans leur maison du Sea Ranch, à 160 kilomètres au nord de San Francisco. Il a développé un troisième axe de sa recherche partitionnelle lors de l'organisation créative de ces séjours, qui ont débuté en 1966 et se sont poursuivis vingt années durant. L'imprégnation forte du lieu était un moteur pour les étudiants participants, et les partitions donnaient un cadre temporel et verbal à des actions d'exploration du territoire par la sculpture, le dessin, la danse, etc.

Ces trois exemples montrent la diversité d'approches qu'Halprin travaille sous l'appellation de "partition ", mais elles n'en sont que fragmentaires. Halprin en effet n'a jamais répété le même système d'écriture d'un projet à l'autre ; ses tentatives sont autant d' "improvisations " autour du mouvement liant l'humain à son environnement. Pour le paysagiste, les partitions n'étaient ni des notations, ni des systèmes, mais de véritables moteurs pour le processus créateur. 
21 Le degré d'ouverture des partitions est l'un des points communs de tous ces essais. En fait, les différentes injonctions et exhortations des partitions se manifestent selon que celles-ci sont ouvertes ou fermées, c'est-à-dire selon que leur auteur laisse plus ou moins de champ libre et de marge de manœuvre à l'interprète. Pour caractériser ses scores, Halprin avait été exploré le champ des partitions dans de multiples domaines, dont la musique. Il a par exemple annoté la partition de l'opéra célèbre de Verdi, La Traviata, en mentionnant les différentes injonctions relevant entre autres de l'environnement (la didascalie situant la scène), de l'activité (ce que fait la chanteuse), de la musique (la partition musicale à proprement parler) ou des indications de temps (les symboles musicaux). Son interprétation orientée d'une notation musicale, qu'on peut qualifier de «traditionnelle », montre l'appropriation qu'il se fait de ce concept de partition.

\section{Mauricio Kagel} classés en quatre catégories : mélodiques, rythmiques, harmoniques et mouvements du corps. Cette partition sous forme de fragments laisse à l'interprète une grande liberté de choix, tant dans le nombre de modèles sélectionnés que dans leur enchaînement ou leur éventuelle simultanéité. Dans un texte d'introduction à la partition, Kagel explique la visée intellectuelle de l'œuvre en listant ses principes de construction et ses conditions de réalisation. Les instrumentistes ont ici un rôle particulier puisqu'en plus de composer librement à partir des matériaux du compositeur, ils sont en charge de la construction de la Machine-orchestre. À ce propos, il est seulement spécifié dans l'introduction que l'on est invité à créer et à faire jouer autant d'instruments sonores que possible, excepté la voix, pour donner à la réalisation l'illusion de la pluralité sonore de l'orchestre. Des exemples de construction de ces machines ont été également insérés dans la partition sous forme de dessins d'Ursula Burghardt, la compagne de Kagel, par ailleurs sculpteur. La construction du système instrumental - ou instrumentarium - et la progression dans la partition (les choix effectués quant aux modèles et aux parties du corps en action) sont faites de concert à partir des schémas 
minutieux des mouvements gestuels dessinés par le compositeur. Les instrumentistes ont parfois réécrit des séquences en rapport avec les instruments qu'ils avaient finalement construits et joués, ce qui prolonge la partition au-delà des modèles mis en place par Kagel.

Cette œuvre bouscule les conventions établies, elle est volontairement provocatrice sur la question de l'orchestre; il faut préciser que l'avènement de l'électroacoustique dans les années 1950 a longtemps fait craindre la disparition de cette institution séculaire qu'est l'orchestre et elle a mobilisé les compositeurs qui se situaient dans ce tournant technologique et conceptuel. L'œuvre de Kagel questionne également la place laissée à l'interprétation, et celle du geste du musicien, qui est ici mis face à une énorme machine, sculpturale, artisanale, mais aussi capable de conditionner les mouvements du corps à la manière des machines industrielles. Les Hommes-orchestre fournissent un effort de concentration intense lors de la création sonore et œuvrent avec une précision qui contraste avec l'apparente liberté qui leur est laissée pour s'engager dans l'écriture et la fabrication de leurs instruments. La partition fournit le matériel musical et les principes de fonctionnement de l'œuvre; c'est ensuite à l'interprète de s'en saisir pour créer un véritable dispositif spatial et scénique très recherché - Kagel qualifie les pièces de cet ordre de «théâtre d'instruments ».

\section{Myriam Gourfink}

Myriam Gourfink est une jeune chorégraphe française qui travaille depuis une dizaine d'années sur l'idée d'une danse liée aux phénomènes résonnants et à leurs conditions de présence dans l'interprétation. Elle s'inscrit dans l'héritage direct de la postmoderne dance - dont on a vu qu'Anna Halprin a fait figure de proue. Le travail de cette chorégraphe permet de mieux comprendre comment, aujourd'hui, ces questions d'écriture par les partitions peuvent être rejouées et mises en chantier.

Tout un pan du travail chorégraphique de Myriam Gourfink questionne en effet la partition. Il faut rappeler que le contexte de la danse ne se prête pas au développement de l'écriture au sens d'une notation écrite. La notation est couramment refusée par les danseurs, la transmission de la danse se faisant majoritairement de façon orale et par monstration des gestes dans un principe d'imitation des présences corporelles. Ce rejet de la notation est motivé par l'idée selon laquelle il faut laisser au mouvement la pleine saveur de sa présence, ce à quoi la notation contreviendrait en figeant le mouvement dansé.

Des tentatives de notation pour la danse ont pourtant vu le jour, notamment celle de Rudolf Laban ${ }^{5}$, qui inspire directement Myriam Gourfink pour son travail de partition. La notation Laban est centrée autour des notions de poids et de dynamiques à l'œuvre dans les actions dites d'effort, qui prennent en compte la réalité des corps et la sensorialité. Mais cette notation, élaborée vers 1920, a certaines limites pour la danse contemporaine aujourd'hui.

Le travail de Myriam Gourfink s'inspire de cette notation en essayant d'outrepasser ces limites qui, selon elle, se situent dans l'appréhension graphique de l'espace comme fini ainsi que dans l'impossibilité de développer une « écriture ouverte », c'est-à-dire " qui laisse la possibilité de choix pour l'interprète ${ }^{6} »$. 

tension différentes temporalités à l'aide des médias danse, musique, vidéo et langage de la partition. Le dispositif de conception pour cette pièce est le suivant: Myriam Gourfink «écrit » d'abord seule cette partition sur l'ordinateur d'après une «vision claire » qu'elle se fait du spectacle; elle la propose aux interprètes du spectacle, qui sont au nombre de sept; elles la déchiffrent ensemble et éventuellement la corrigent ou la précisent ; puis, lors du spectacle, la chorégraphe modifie des éléments de cette partition en temps réel grâce à des procédés technologiques ; ces partitions sont lues et jouées par les danseuses sur scène. La modification s'effectue en fonction de l'espace que les danseuses dessinent sur le plateau. Les écrans projetés à chacune des danseuses sont divisés horizontalement en deux: Myriam Gourfink applique des contraintes, situées sur la moitié supérieure de l'écran, à des actions, situées sur la moitié inférieure de l'écran. Ces actions qui évoluent lentement au fil de la pièce ont trait aux parties du corps à mobiliser, et à la façon de les mobiliser. Les modifications concernent également les déplacements sur la scène et des indications temporelles comme des répétitions.

31 La partition est pour Myriam Gourfink un outil d'écriture puisqu'elle participe pleinement à la composition de ses pièces ; mais elle est aussi un outil de lecture, lui servant à affiner son analyse d'autres chorégraphies par exemple. Elle laisse une part d'ouverture, à la fois pour les interprètes, qui n'auront jamais à effectuer deux fois le même spectacle, et pour la chorégraphe qui, à chaque répétition ou représentation, peut composer de nouveau.

À la question de l'improvisation, Myriam Gourfink répond qu'elle préfère, dans son travail, parler de "partitions ouvertes, comme dans le champ musical des années 1970 »; elle avance qu'il pourrait à la limite s'agir d'une « improvisation extrêmement structurée ${ }^{8}$ ». Mais l'improvisation en danse est avant tout basée sur une tâche à mémoriser, ce qui n'est pas le cas ici. Les interprètes se meuvent très lentement - c'est un parti pris esthétique de la chorégraphe - selon de très petites précisions qui ne sont pas mémorisables car infimes, et selon une dramaturgie prédéfinie. La pensée de Myriam Gourfink se matérialise dans l'écriture qui obéit à un principe de composition, mais elle laisse la place à l'improvisation des danseuses pour créer une œuvre qui n'est jamais chaque fois tout à fait la même.

\section{Vers l'œuvre ouverte}

Ces trois dispositifs, bien qu'ils appartiennent à des champs différents, présentent un certain nombre de questionnements similaires qu'ils essaient de résoudre chacun à leur manière et selon la culture historique qui prédétermine leur discipline.

On remarquera d'abord qu'Halprin comme Kagel et Gourfink ont une approche multidisciplinaire de leur domaine qui tend à croiser les champs créatifs pour provoquer de savants dispositifs. Ainsi, Halprin fait appel à la danse et à la scénographie, mais également à la psychologie gestaltiste ; Kagel s'approche du théâtre pour faire jouer ses instruments, mais aussi du savoir-faire artisanal; et Myriam Gourfink mêle danse, vidéo et musique en une forme non seulement visuelle, mais également écrite à travers ses partitions. Le contexte des années 1960 y est pour beaucoup dans ces « mélanges $^{9}$ » de disciplines devenues aujourd'hui classiques. 
contexte crée également la notion de "partitions ouvertes ", que corroborent ces trois artistes et qui n'est pas sans rappeler L'Euvre ouverte d'Umberto Eco, paru en 1962. Le philosophe italien y fait état d'une nouvelle poétique de «l'œuvre ouverte ", qui existe aussi bien dans le champ littéraire ou plastique que dans celui de la musique. Au cœur de ces «œuvres ouvertes» ou «œuvres en mouvement» se situent les interprètes, qui entretiennent une relation particulière avec ce qu'ils voient, entendent, perçoivent ou parcourent: «En somme, l'auteur offre à l'interprète une œuvre à achever. Il ignore de quelle manière précise elle se réalisera, mais il sait qu'elle restera son œuvre; au terme du dialogue interprétatif, se concrétisera une forme organisée par un autre, mais une forme dont il reste l'auteur. Son rôle consiste à proposer des possibilités déjà rationnelles, orientées et dotées de certaines exigences organiques qui déterminent leur développement ${ }^{10}$." L'analyse d'Umberto Eco permet de faire comprendre ce nouveau positionnement artistique qui vise à ouvrir ses dispositifs de création à l'interprétation, tout en assumant le rôle essentiel du "compositeur" comme auteur de son œuvre. Nous avons pu voir en effet dans les trois formes étudiées - celle d'un paysagiste, celle d'un compositeur et celle d'une chorégraphe - le rôle donné à l'ouverture dans l'écriture de la partition. L'improvisation a lieu à travers la présence du danseur, le rôle du musicien et la mise en action ou en mouvement de l'espace qui est sciemment mise en œuvre dans la composition. Les deux processus sont donc intrinsèquement liés; ils élaborent dans l'acte de création divers regards interprétatifs susceptibles à tout moment d'évoluer et de faire évoluer l'objet duquel ils se saisissent.

Le champ d'ouverture de la question de l'interprétation en rapport avec des possibilités d'écriture peut être pertinent et approprié aux questionnements du paysagiste qui, en créant une mise en espace par la composition, interprète à la fois le paysage tel qu'il s'offre à ses yeux et les regards qui pourront se poser sur ce paysage ${ }^{11}$. Son écriture est donc une invitation à l'improvisation; il s'agit de donner des possibilités infinies de mouvements par une structure que l'on voudrait « ouverte ».

Dans ce cadre, la partition semble être l'un des outils de création envisageable pour dépasser ces contradictions apparentes entre composition et improvisation qui donneraient à la première le pouvoir de la structure et à la seconde la toute-puissante liberté. Les écritures que nous avons découvertes s'inscrivent délibérément dans une recherche de structures et de passages entre le déterminé et l'indéterminé : les degrés d'ouverture mis en œuvre par Halprin dans les cycles RSVP, les possibilités de «construction» offertes à l'interprète par Kagel, ou encore les interactions perpétuelles entre auteure et interprètes chez Gourfink. Ces jeux entre composition et improvisation, rendus visible par la partition comme outil de création, sont ouverts à bien d'autres écritures. Car, comme le dit Laurence Louppe, qui écrit sur la danse : « La partition n'a pas de bords. Elle est extensive au point que tout événement concomitant ou non, lointain ou proche, visible ou invisible, l'infiltre, l'affecte, et la réoriente à chaque instant ${ }^{12}$.» Ainsi, l'exploration de ces univers très différents et pourtant si proches dans leurs questionnements saura peut-être intriguer la conception en paysage en lui offrant des manières de renouveler ses modes de représentation traditionnels pour lier l'improvisation à la composition.

Projets de paysage, 8 | 2012 


\section{BIBLIOGRAPHIE}

Halprin, A., Mouvements de vie. 60 ans de recherches, de créations et de transformations par la danse, Bruxelles, Contredanse, traduit par Élise Argaud et Denise Luccioni, 2009.

Halprin, L., The RSVP cycles: creative processes in the human environment, New York, G. Braziller, 1970.

Halprin, L., Notebooks, 1959-1971, Cambridge, MIT Press, 1972.

Halprin, L. (avec Burns, J.), Taking part: a workshop approach to collective creativity, Cambridge, MIT Press, 1974.

Halprin, L., The Sea Ranch: Diary of an idea, Berkeley, Spacemaker Press, 2002.

Kagel, M., Tam-Tam. Monologues et dialogues sur la musique (1975), Paris, Christian Bourgois, 1983.

Kassel, M., Mauricio Kagel, Zwei-Mann-Orchester, Essays und Dokumente, Basel, Paul Sacher Stiftung, Schwabe Verlag, 2011.

Pousin, F., « De la chorégraphie à l'architecture du paysage: noter pour concevoir ", Les Carnets du paysage, $\mathrm{n}^{\circ}$ 13-14, Comme une danse, Arles/Versailles, Actes Sud/ENSP, 2007.

Tiberghien, G., « Lawrence Halprin: danse et mouvement du monde », dans Les Carnets du paysage, $\mathrm{n}^{\circ}$ 13-14, Comme une danse, Arles/Versailles, Actes Sud/ENSP, 2007.

Collectif, De l'une à l'autre. Composer, apprendre et partager en mouvements, Bruxelles, Contredanse, 2010.

Web

Image d'une œuvre $\mathrm{n}^{\circ} 6$ : «Les temps tiraillés », série documentaire publiée par l'Ircam, URL : https://medias.ircam.fr/x895ac7_images-dune-uvre-n-6-les-temps-tirail.

\section{NOTES}

1. Pouillaude, F., « Vouloir l'involontaire et répéter l'irrépétable », dans Boissière , A. et Kintzler, C., Approche philosophique du geste dansé, Villeneuve d'Ascq, Presses du Septentrion, 2006, p. $145-161$.

2. «Cartographier, construire, inventer - Notes pour une épistémologie de la démarche de projet ", Chap. IV de l'ouvrage Le Goût du monde. Exercices de paysage, Arles, Actes Sud, 2009, p. 141-188.

3. Sur la musique américaine au tournant des années 1950, voir l'ouvrage de M. Nyman écrit en bout de course de ce mouvement en 1974, Experimental Music. Cage et au-delà, trad. Nathalie Gentili, avant-propos Brian Eno Paris, Allia, 2005.

4. On peut voir un extrait de quelques minutes de la re-création de la pièce à Basel en avril 2011 en ligne sur http://www.youtube.com/watch?v=oM5SttMyulE.

5. Pour découvrir la notation Laban, on pourra lire l'ouvrage du danseur, La Maîtrise du mouvement, édité chez Actes sud (1994); et pour plus de clarté, l'interprétation qu'en donne Jacqueline Challet-Haas dans Grammaire de la notation Laban. Cinétographie Laban, Pantin, Centre national de la danse, coll. « Cahiers de la pédagogie », 1999, vol. I.

6. Interview de Myriam Gourfink par Baptiste Andrien dans De l'une à l'autre. Composer, apprendre et partager en mouvements, Bruxelles, Contredanse, 2010, p. 189-197 
7. Un extrait de la captation du spectacle est visible en ligne sur https://www.youtube.com/ watch?v=iedgRsGxWXU.

8. Interview de la chorégraphe donnée sur dancetech.tv, visible en ligne sur http:// www.youtube.com/watch?v=Q20r7Lj2OBU\&feature=related.

9. Ce mot est utilisé par le danseur Steve Paxton à propos des pratiques caractérisant le champ de l'art dans les années 1960. Dans De l'une à l'autre, op. cit., p. 60.

10. Eco, U., L'Euvre ouverte, Paris, Seuil, coll. « Points Seuil », 1962, p. 34.

11. Le vocabulaire « visuel » que j'emploie ici est essentiellement métaphorique ; car ce n'est bien entendu pas seulement la vue qui agit sur et dans le paysage, mais également les présences physiques (les façons de se promener, de sentir, d'écouter, de s'orienter, etc.).

12. Louppe, L., Poétique de la danse contemporaine. La suite, Bruxelles, Contredanse, 2007, chap. «Partitions », p. 23.

\section{RÉSUMÉS}

La conception en paysage se trouve confrontée à la difficulté d'écrire une matière dont la présence est en perpétuel mouvement. Ce problème questionne également, entre autres, les disciplines de la danse et de la musique. L'un des outils d'écriture de cette dernière est la partition, dont le rôle est traditionnellement dévolu à la composition. Mais dans la seconde moitié $\mathrm{du} \mathrm{xx}^{\mathrm{e}}$ siècle, sous l'impulsion donnée par les "œuvres ouvertes », apparaissent des partitions qui cherchent à lier composition et improvisation. Ces recherches influenceront le paysagiste américain Lawrence Halprin, qui travaillera sur les scores pour la conception urbaine et paysagère. À travers l'étude de trois dispositifs créatifs - Lawrence Halprin en paysage, Mauricio Kagel en musique, et Myriam Gourfink en danse - nous posons la question de cette tension entre composition et improvisation comme ouverture au mouvement.

The conception in landscape is confronted to the difficulty to write a material which is in a perpetual motion. This issue tackles also, among other things, the disciplines of dance and music. One of the writing tools of the latter is the score - the role of which is devolved to composing. But in the second half of the $20^{\text {th }}$ century, and following the impetus of the "open works", some music scores willing to link composition and improvisation have appeared. These researches will influence the American landscaper Lawrence Halprin who will work on scores for the landscape and urban conception. Through the study of three creative devices - Lawrence Halprin in landscape, Mauricio Kagel in music, and Myriam Gourfink in dance - the issue of tension between composition and improvisation opened to motion is raised.

\section{INDEX}

Mots-clés : processus de conception, danse, musique, œuvre ouverte, compositionimprovisation

Keywords : conception process, dance, music, open work, composition-improvisation 


\section{AUTEUR}

\section{MATHILDE CHRISTMANN}

Mathilde Christmann est doctorante (deuxième année) au LACTH (Laboratoire conception territoire histoire) de l'École d'architecture et de paysage de Lille, sous la direction de Catherine Grout, en codirection avec Anne Boissière du Centre d'étude des arts contemporains, Lille 3. mathilde.christmann[at]yahoo[dot]fr 